Case Report

\title{
Short Interval Infield Sarcoma Development following Resection of Glioblastoma and Adjuvant Radiotherapy and Temozolomide
}

\author{
Fahad E. Alotaibi and Kevin Petrecca \\ Department of Neurology and Neurosurgery, Montreal Neurological Institute and Hospital, \\ McGill University and Department of Neurosurgery, McGill University Health Centre, 3801 University Avenue, \\ 109 Montreal, QC, Canada H3A 2B4 \\ Correspondence should be addressed to Kevin Petrecca; kevin.petrecca@mcgill.ca
}

Received 24 June 2013; Accepted 24 July 2013

Academic Editor: Lothar Bergmann

Copyright (C) 2013 F. E. Alotaibi and K. Petrecca. This is an open access article distributed under the Creative Commons Attribution License, which permits unrestricted use, distribution, and reproduction in any medium, provided the original work is properly cited.

Background. The development of 2 unassociated brain cancers in the same patient is a rare occurrence. Secondary cancers are generally thought to develop as an oncogenic consequence of the radiation therapy delivered to treat the primary cancers, always requiring a significant time interval between radiation treatment and secondary cancer development. Case Description. We report the development of an undifferentiated myxoid sarcoma only 13 months following radiation therapy for a glioblastoma. Conclusion. This case represents the shortest time interval reported between radiation therapy and secondary brain cancer development.

\section{Introduction}

A well-established long-term complication of cranial radiation is the development of secondary tumors. These tumors are typically meningiomas, but gliomas, ependymomas, medulloblastomas, sarcomas, schwannomas, and primitive neuroectodermal tumors have been reported [1]. Most commonly, a long interval is required between the time of radiation delivery and the appearance of the second tumor, often decades in the case of meningiomas. We report the development of an undifferentiated myxoid sarcoma only 13 months following radiation therapy and temozolomide for a glioblastoma.

\section{Case Description}

A 47-year-old man presented with a seizure. Magnetic resonance imaging (MRI) revealed a lesion involving the right temporal lobe that was hyperintense on $\mathrm{T}_{2}$-weighted sequences with a small area of gadolinium enhancement, consistent with a malignant glioma (Figures 1(a) and 1(b)). The patient had no personal cancer history and no family history of a cancer syndrome. He underwent resection
(Figure 1(c)) and the lesion was classified as a World Health Organization glioblastoma (GBM). The cancer exhibited hypermethylation at the $\mathrm{O}^{6}$-methylguanine-DNA methyltransferase promoter region, was wild-type at the IDH 132 position, and the epidermal growth factor receptor was amplified. Adjuvant therapy consisted of radiotherapy (60 Gy) and chemotherapy according to the Stupp protocol [2]. Maintenance temozolomide treatment continued for 12 months.

One month later, a surveillance MRI revealed a new lesion within the resection cavity and extending over the right frontal convexity that appeared to be contained within the subdural extraleptomeningeal space (Figures 2(a)-2(d)). The lesion, which was iso- to hypointense on $\mathrm{T}_{2}$-weighted sequences and homogenously enhancing on gadoliniumenhanced $\mathrm{T}_{1}$-weighted sequences, was consistent with a hematoma or an atypical recurrent GBM. There was no evidence of recurrent GBM within the brain. Repeat imaging 2 months later demonstrated progression of the lesion over the entire right hemisphere (Figures 2(e)-2(h)).

A second craniotomy was performed which revealed a solid, firm tumor within the subdural and extraleptomeningeal space. A clear dissection plane existed 


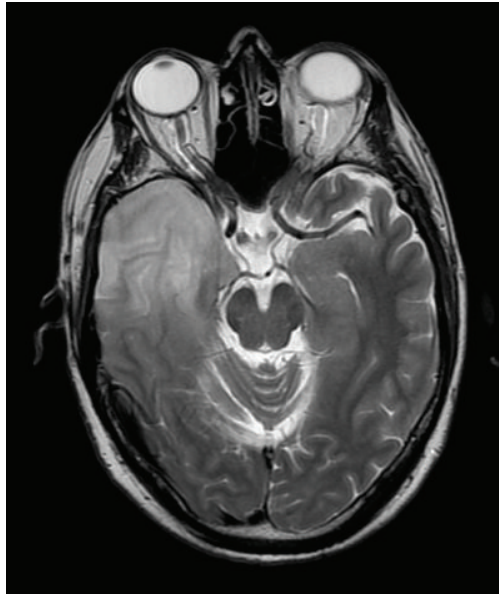

(a)

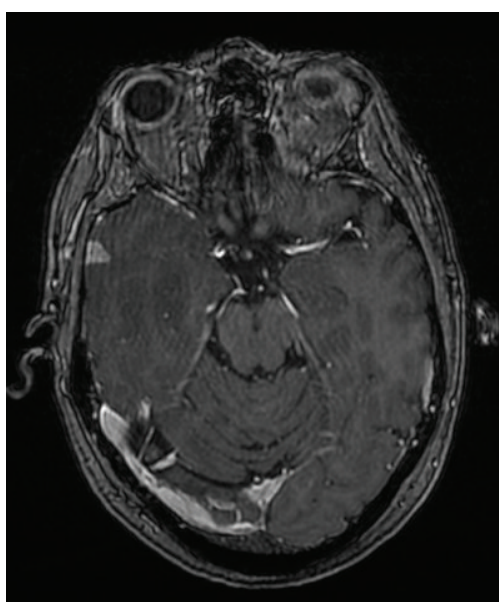

(b)

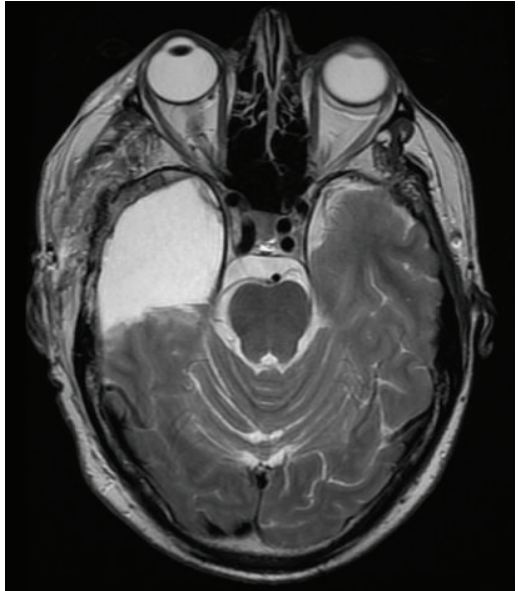

(c)

Figure 1: Pre- and Post-Operative Glioblastoma Imaging. (a) Pre-operative axial $\mathrm{T}_{2}$-weighted image. (b) Pre-operative gadolinium-enhanced $\mathrm{T}_{1}$-weighted image. (c) Post-operative axial $\mathrm{T}_{2}$-weighted image.

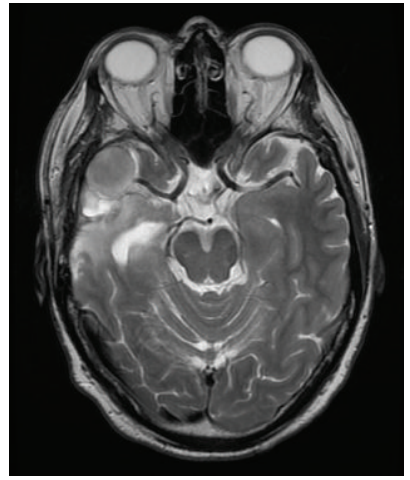

(a)

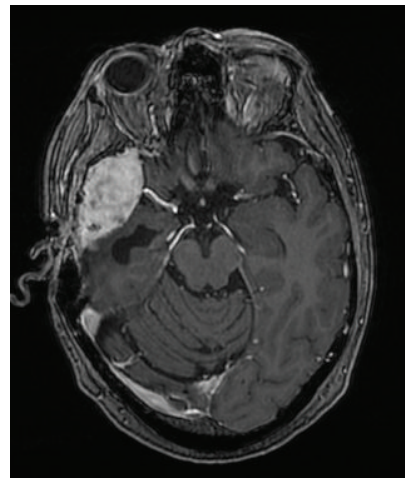

(e)

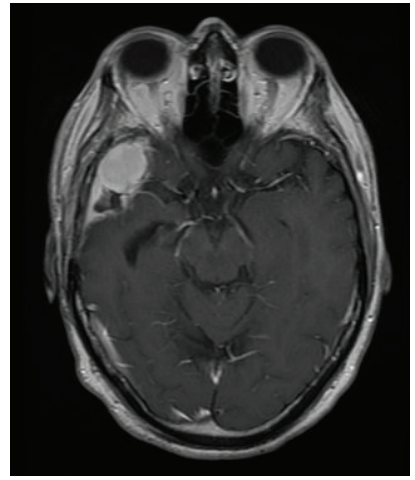

(b)

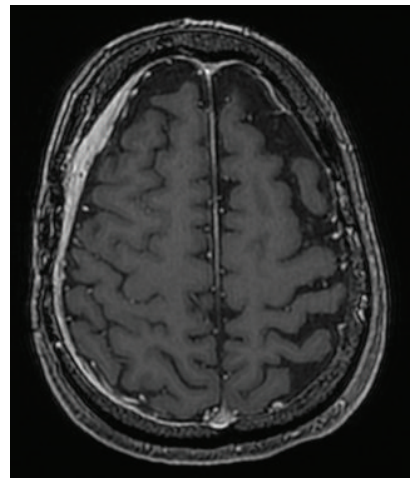

(f)

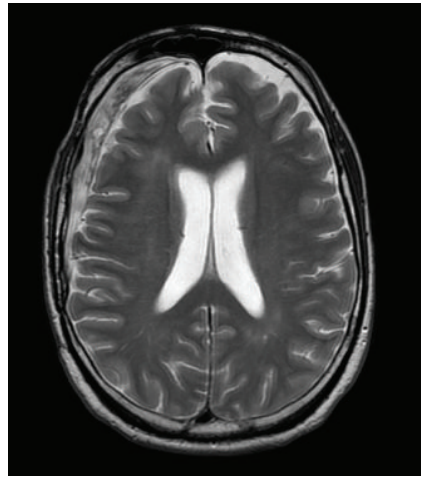

(c)

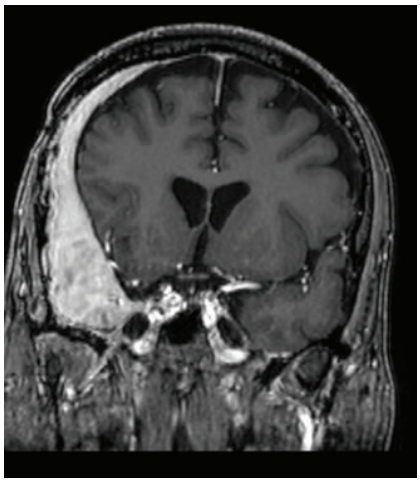

(g)

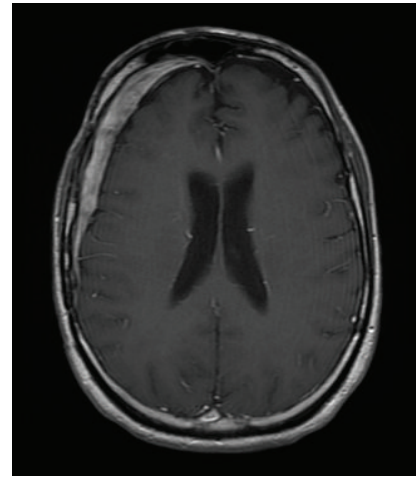

(d)

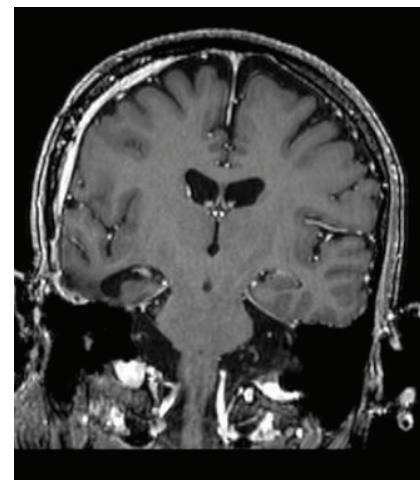

(h)

FIGURE 2: Appearance and progression of secondary tumor. $\mathrm{T}_{2}$-weighted ((a) and (c)) and gadolinium enhanced $\mathrm{T}_{1}$-weighted ((b) and (d)) images demonstrating the first appearance of the secondary tumor. ((e)-(h)) Gadolinium enhanced $\mathrm{T}_{1}$-weighted images showing progression of secondary tumor.

between the tumor and the pia-arachnoid (Figure 3(a)), and a complete resection was achieved (Figure 3(b)). This tumor was classified as an undifferentiated myxoid sarcoma based on the lack of a glial proliferation, strong CD68 and vimentin immunolabeling in the majority of tumor cells and the absence of CDla and GFAP immunolabeling. The patient went on to receive radiotherapy to the resection margins followed by doxorubicin chemotherapy. An MRI performed 3 months following the second surgery revealed no evidence of recurrent sarcoma (Figures 3(c)-3(f)). 


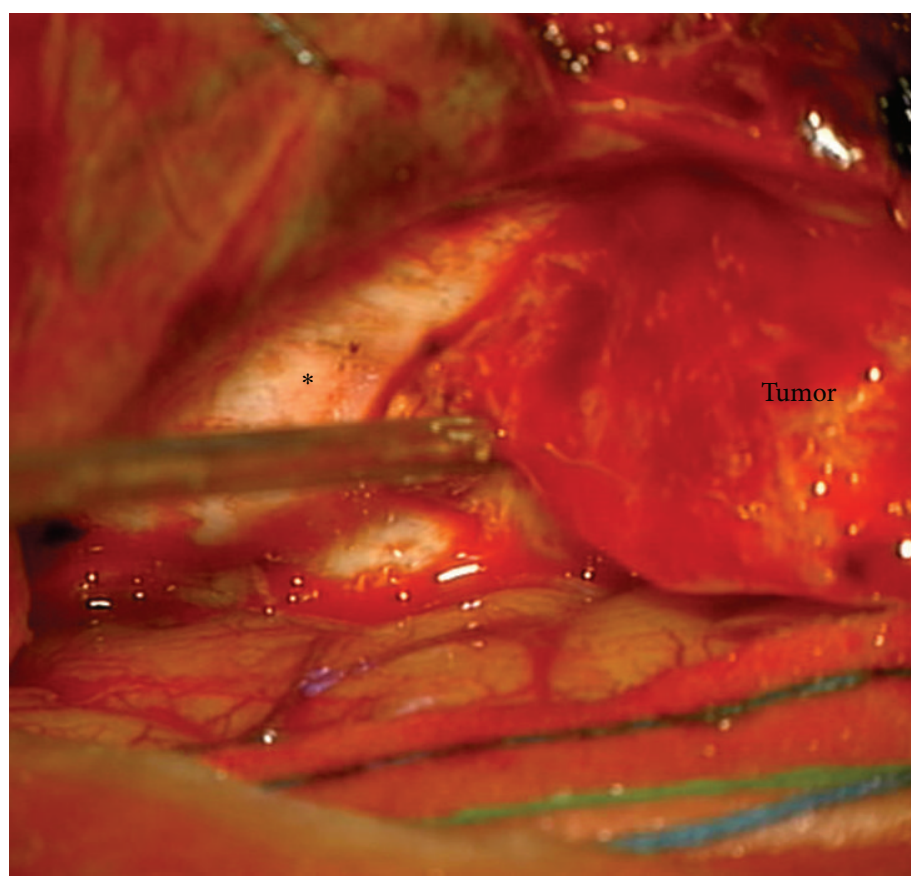

(a)

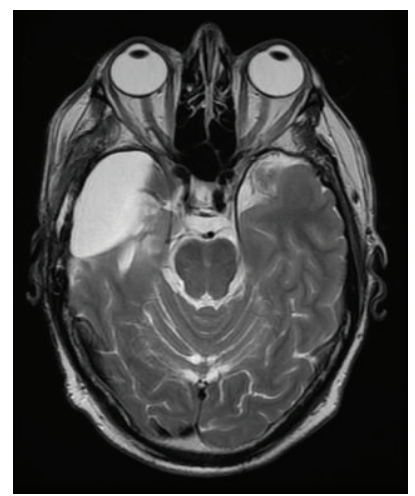

(c)

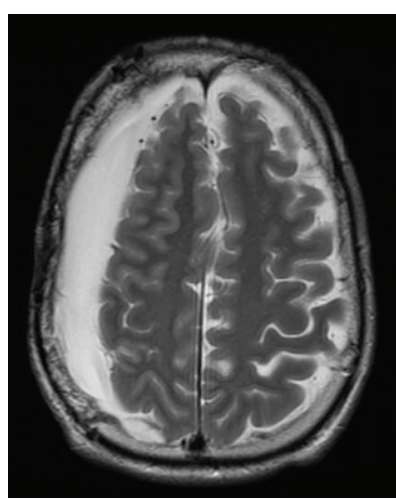

(d)

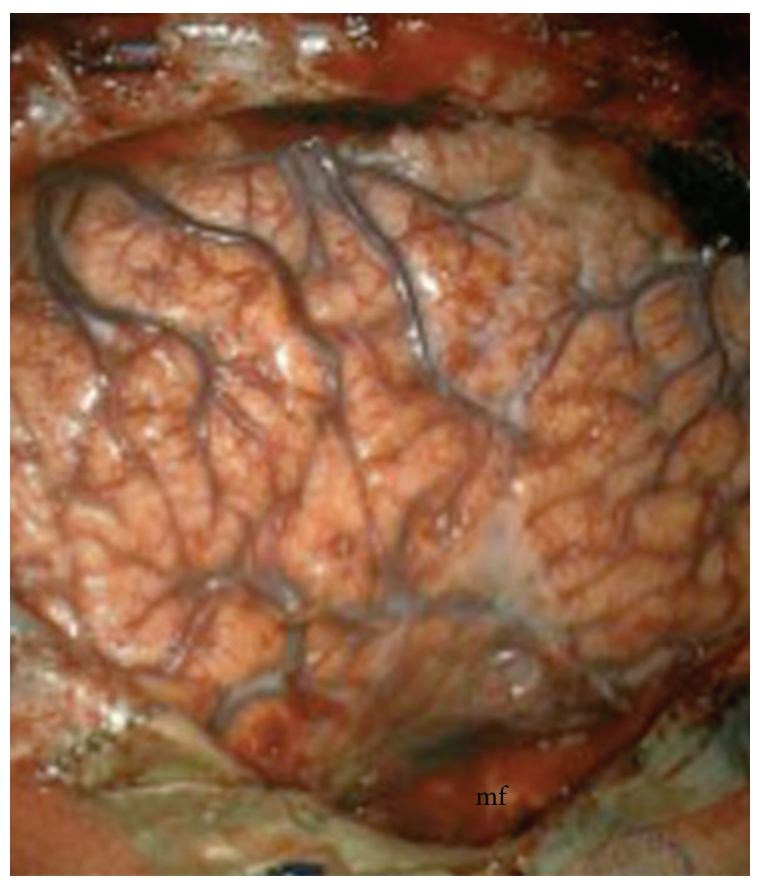

(b)

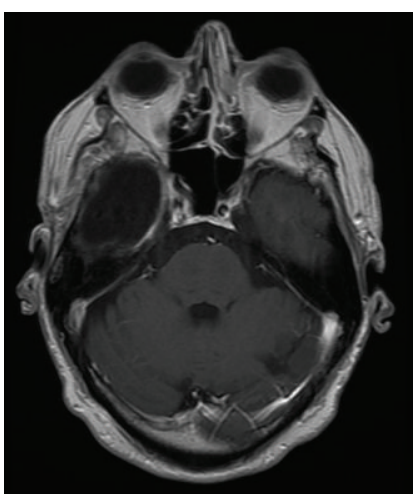

(e)

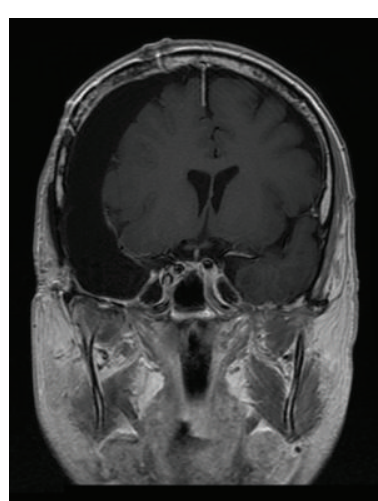

(f)

FIGURE 3: Intraoperative findings and postoperative results. (a) Intraoperative image showing subdural extra-leptomeningeal tumor. Asterisk indicates anterior fossa dura; $\mathrm{mf}$ : middle fossa. (b) Intraoperative image revealing complete resection. $\mathrm{T}_{2}$-weighted ((c) and (d)) and gadolinium enhanced $\mathrm{T}_{1}$-weighted ((e) and (f)) images showing no evidence of sarcoma recurrence at 3 months.

\section{Discussion}

Tumors that have been reported to develop following cranial radiation include meningiomas, gliomas, sarcomas, and medulloblastomas [1-17]. The time interval between radiotherapy and the occurrence of these tumors ranges from 4 to 47 years, with a mean interval of 18.8 years. Chowdhary et al. [1] reported a significant positive correlation between the time interval following radiotherapy and the development of a meningioma (21.8 years) or a sarcoma (7.7 years).

The coincidence of a GBM and an intracranial sarcoma in the same patient is rare; only 2 cases have been reported. A 9-month-old with a GBM treated with surgery and chemotherapy, but not radiotherapy, developed an intracranial fibrosarcoma 5 years later [7]. The second, a 43-year-old with an intraparenchymal fibrosarcoma treated with surgery followed by fractionated conformal radiotherapy developed a recurrence 3 months later and was treated with stereotactic radiosurgery. Nine months later the lesion progressed and a second surgery was performed. The lesion was classified as a GBM [18].

One case of intracranial myxoid sarcoma has been reported, a metastasis from primary lung lesion [19]. There are no reports suggesting an association between temozolomide therapy and the development of myxoid sarcomas.

The case described here is the shortest time interval reported between the completion of radiotherapy (13 months) and the development of a second malignancy. This short latency suggests that prior radiotherapy may not be the cause of this undifferentiated myxoid sarcoma. An alternate hypothesis is that this patient carries an underlying genetic disorder predisposing to cancer development. 


\section{References}

[1] A. Chowdhary, A. M. Spence, L. Sales, R. C. Rostomily, J. K. Rockhill, and D. L. Silbergeld, "Radiation associated tumors following therapeutic cranial radiation," Surgical Neurology International, vol. 3, p. 48, 2012.

[2] R. Stupp, W. P. Mason, M. J. van den Bent et al., "Radiotherapy plus concomitant and adjuvant temozolomide for glioblastoma," The New England Journal of Medicine, vol. 352, no. 10, pp. 987-996, 2005.

[3] D. W. Dodick, B. Mokri, E. G. Shaw, G. M. Miller, and K. K. Unni, "Sarcomas of calvarial bones: rare remote effect of radiation therapy for brain tumors," Neurology, vol. 44, no. 5, pp. 908-912, 1994.

[4] F. T. Garner, D. M. Barrs, D. M. Lanier, T. E. Carter, and R. E. Mischke, "Radiation-induced sarcoma of the skull: a case report," Otolaryngology, vol. 99, no. 3, pp. 326-329, 1988.

[5] J. M. Gomona and A. Shaked, "Radiation induced meningiomas," Neuroradiology, vol. 23, no. 4, pp. 211-212, 1982.

[6] J. C. Gonzalez Vitale, R. E. Slavin, and J. D. McQueen, "Radiation-induced intracranial malignant fibrous histiocytoma," Cancer, vol. 37, no. 6, pp. 2960-2963, 1976.

[7] J. M. Kaminski, C.-C. Yang, F. Yagmai, B. Movsas, M. Lee, and J. T. Barrett, "Intracranial fibrosarcoma arising 5 years after chemotherapy alone for glioblastoma multiforme in a child," Pediatric Neurosurgery, vol. 33, no. 5, pp. 257-260, 2000.

[8] P. P. Kumar, R. R. Good, F. M. Skultety et al., "Radiation-induced neoplasms of the brain," Cancer, vol. 59, no. 7, pp. 1274-1282, 1987.

[9] M. L. C. Maat-Schieman, T. A. M. Bots, T. W. M. Thomeer, and G. J. Vielvoye, "Malignant astrocytoma following radiotherapy for craniopharyngioma," The British Journal of Radiology, vol. 58, no. 689, pp. 480-482, 1985.

[10] F. J. Mark, J. Poen, L. M. Tran, Y. S. Fu, M. T. Selch, and R. G. Parker, "Postirradiation sarcomas: a single-institution study and review of the literature," Cancer, vol. 73, pp. 2653-2662, 1994.

[11] G. Marus, C. V. Levin, and G. S. Rutherfoord, "Malignant glioma following radiotherapy for unrelated primary tumors," Cancer, vol. 58, no. 4, pp. 886-894, 1986.

[12] J. Munk, E. Peyser, and J. Gruszkiewicz, "Radiation induced intracranial meningiomas," Clinical Radiology, vol. 20, no. 1, pp. 90-94, 1969.

[13] I. J. Rimm, F. C. Li, N. J. Tarbell et al., "Brain tumors after cranial irradiation for childhood acute lymphoblastic leukemia. A 13year experience from the Dana-Farber Cancer Institute and The Children's Hospital," Cancer, vol. 59, no. 8, pp. 1506-1508, 1987.

[14] E. Robinson, A. I. Neugut, and P. Wylie, "Clinical aspects of postirradiation sarcomas," Journal of the National Cancer Institute, vol. 80, no. 4, pp. 233-240, 1988.

[15] A. B. Rubinstein, M. N. Shalit, M. L. Cohen et al., "Radiationinduced cerebral meningioma: a recognizable entity," Journal of Neurosurgery, vol. 61, no. 5, pp. 966-971, 1984.

[16] R. E. Scully, E. J. Mark, W. F. McNeely, and B. U. McNeely, "Case records of the Massachusetts General Hospital: case 26-1988," The New England Journal of Medicine, vol. 318, pp. 1742-1750, 1988.

[17] D. Soffer, S. Pittaluga, M. Feiner, and A. J. Beller, "Intracranial meningiomas following low-dose irradiation to the head," Journal of Neurosurgery, vol. 59, no. 6, pp. 1048-1053, 1983.

[18] A. O. Adeleye, Y. Fellig, F. Umansky, and Y. Shoshan, "Rapid growth of primary cerebral fibrosarcoma with conversion to glioblastoma at second recurrence," Journal of Neuro-Oncology, vol. 92, no. 2, pp. 233-238, 2009.

[19] K. Thway, A. G. Nicholson, K. Lawson et al., "Primary pulmonary myxoid sarcoma with EWSR1-CREB1 fusion: a new tumor entity," American Journal of Surgical Pathology, vol. 35, no. 11, pp. 1722-1732, 2011. 


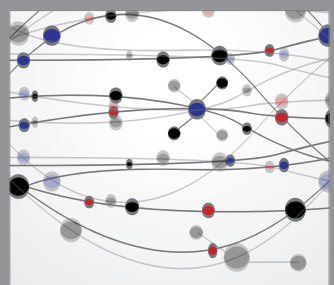

The Scientific World Journal
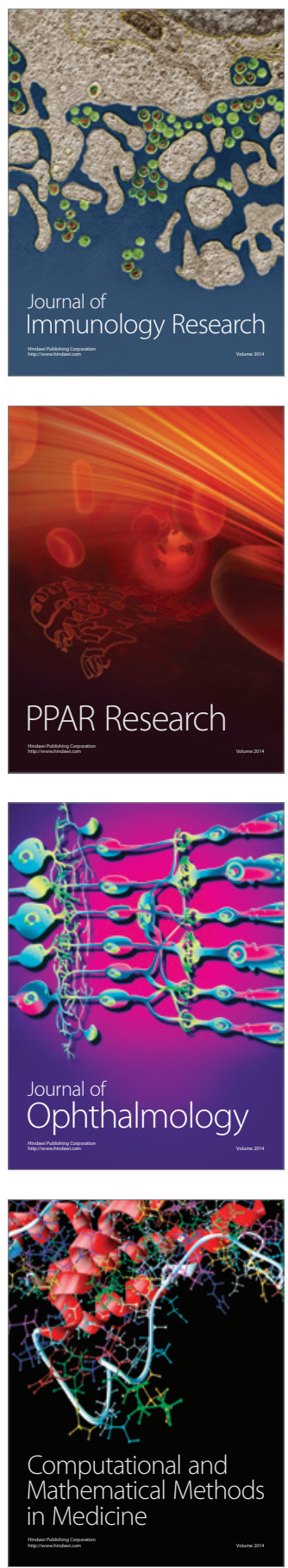

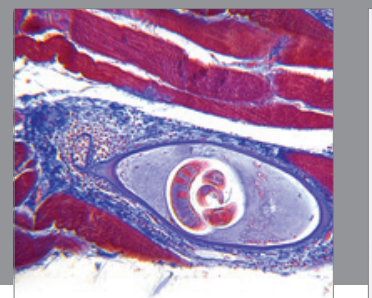

Gastroenterology

Research and Practice
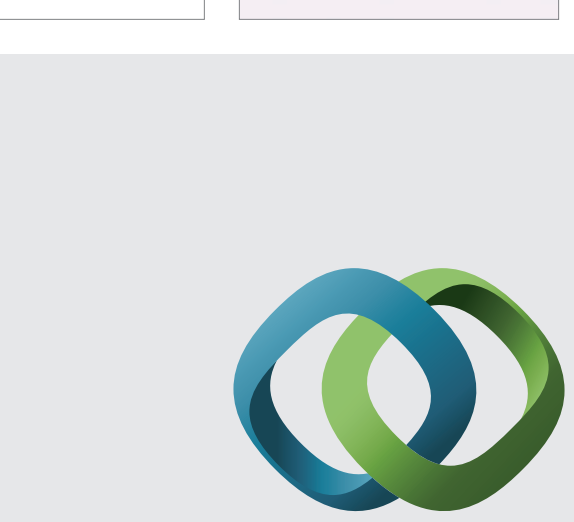

\section{Hindawi}

Submit your manuscripts at

http://www.hindawi.com
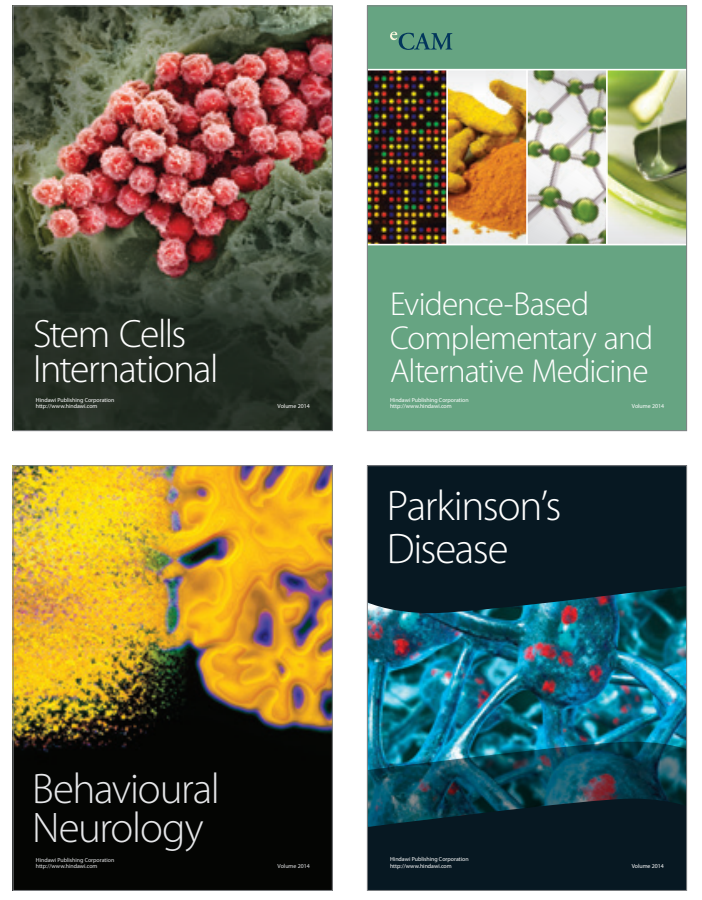
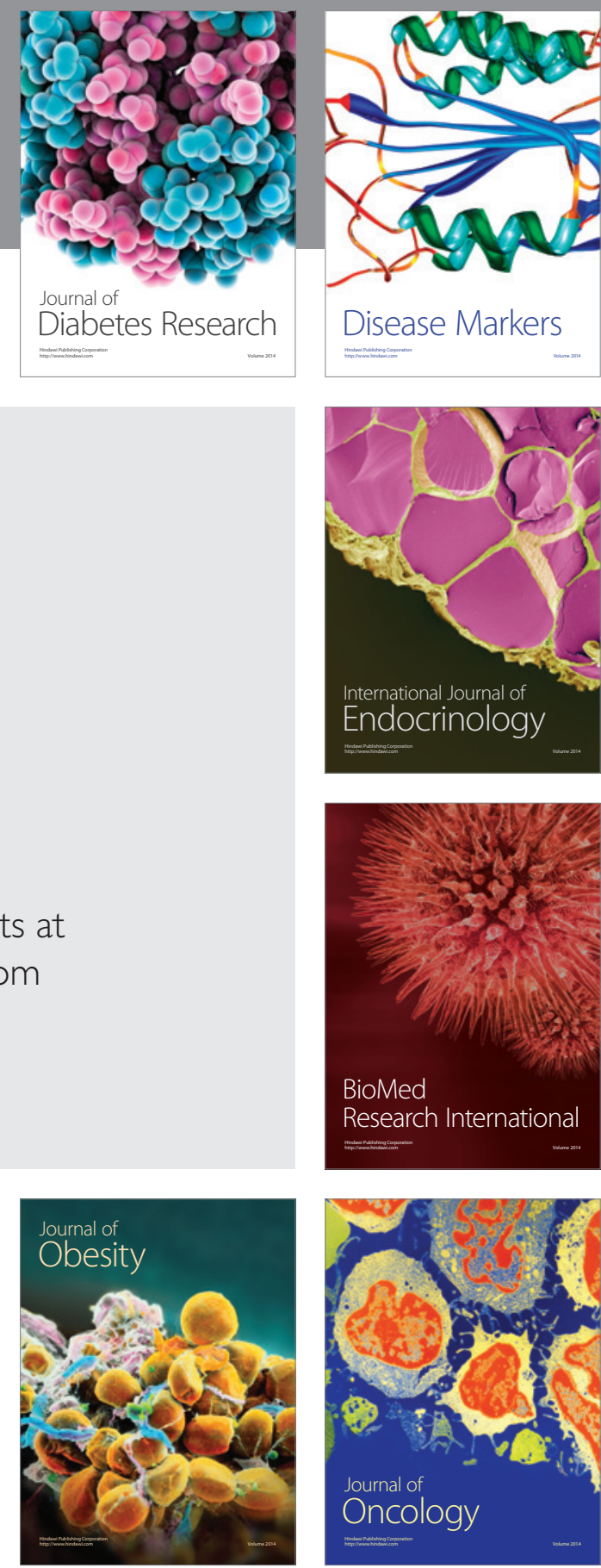

Disease Markers
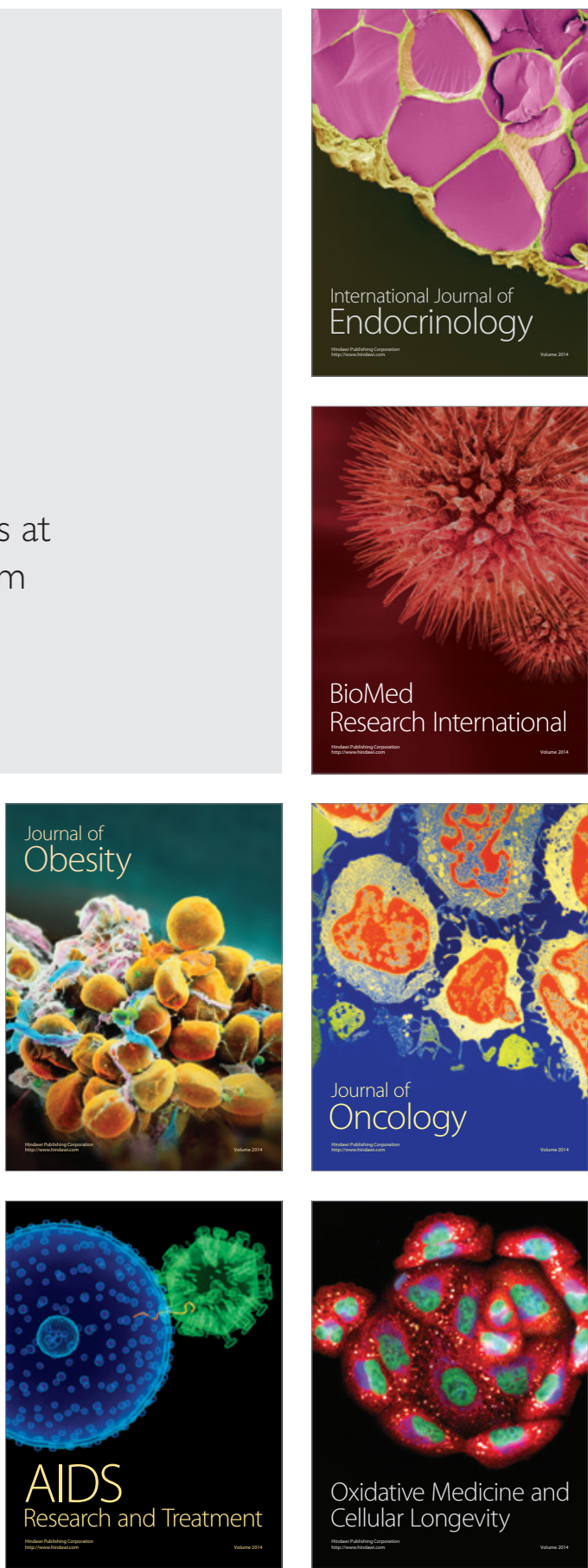